\title{
Synthesis and characterization of La and Mo co-doped strontium titanate nanomaterials for intermediate temperature solid oxide fuel cell anode
}

\author{
Saurabh Singh\#, Vani Pawar, S. K. Mandal and Prabhakar Singh* \\ Department of Physics, Indian Institute of Technology (BHU), Varanasi-221005, India \\ Email: *psingh.app@iitbhu.ac.in; \#ssingh.rs.app13@iitbhu.ac.in
}

Solid Oxide Fuel Cells (SOFCs) are one of the most attractive electrochemical energy conversion systems, as these devices present a clean energy production, thus promising high efficiencies and low environmental impact [1]. The electrodes are the main components that decisively control the performance of a SOFC. Conventional, anode materials (like Ni-YSZ) are operates at very high temperature. Therefore, cost-effective materials which operate at relatively lower temperatures are still required [2]. In present study, we have synthesized $\mathrm{La}$ and Mo co-doped A-site deficient Strontium Titanate (LSMT) via Chemical reaction route [2]. The particle diameters of LSMT powders calcined at 1000 ${ }^{\circ} \mathrm{C}$ are all less than $60 \mathrm{~nm}$. The LSMT pellet sintered in air at $1400^{\circ} \mathrm{C}$ for $6 \mathrm{~h}$ shows a highly densified microstructure composed of polyhedral grains on a micron scale.

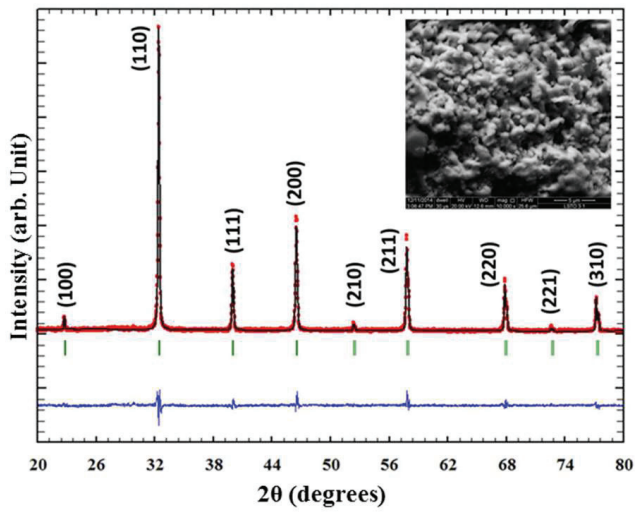

Figure 1: X-ray Rietveld refinement of LSMT samples, Inset depicts SEM micrograph of the system

The structural, compositional, microstructural and density of the pellet have been investigated employing XRD, XPS, SEM and Archimedes Principle, respectively. Figure 1 shows XRD pattern of LSMT samples with Rietveld refinement. Surface area and porosity of the compositions have been determined via BET Surface area analyzer.

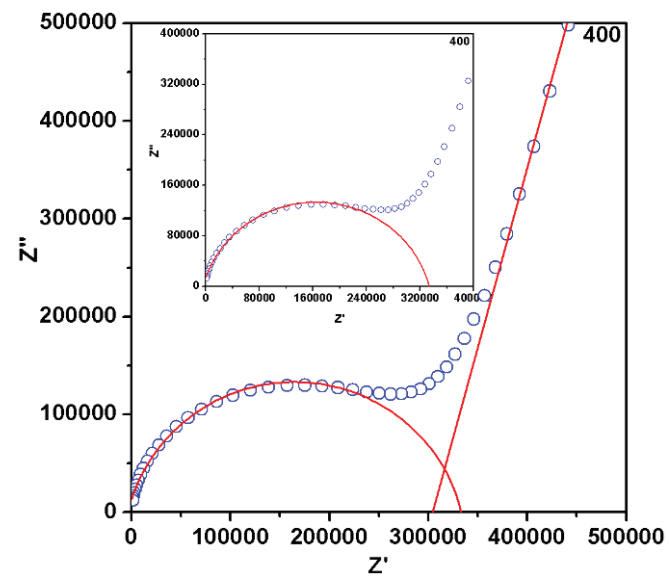

Figure 2: The complex plane Impedance plot of LSMT system

The electrical conductivity of the systems has been determined by impedance spectroscopy techniques (Figure 2). The electrical conductivity of the 30 mole $\%$ doped $\mathrm{La}$ Strontium Titanate with 7 mole \% doped Mo has been found to be $3.8 \times 10^{-3} \mathrm{~S} \mathrm{~cm}^{-1}$ at $600{ }^{\circ} \mathrm{C}$.

Keywords: IT-SOFC, LSMT, impedance spectroscopy, electrical conductivity

\section{References}

1. X. Zhou, N. Yan, K. T. Chuang and J. Luo, RSC Adv., 4 (2014), 118-131.

2. U. Balachandran and N. G. Eror, J. Electrochem. Soc. 129 (1982), 1021-1026. 